\title{
ON THE GENUS OF GRAPHS WITH LICK-WHITE NUMBER $k$
}

\author{
JOHN MITCHEM
}

\begin{abstract}
A graph is called $n$-degenerate if each of its subgraphs has a vertex of degree at most $\boldsymbol{n}$. For each $\boldsymbol{n}$ the Lick-White number of graph $\boldsymbol{G}$ is the fewest number of sets into which $V(G)$ can be partitioned such that each set induces an $n$-degenerate graph. An upper bound is obtained for the Lick-White number of graphs with given clique number. A number of estimates are derived for the number of vertices in triangle-free graphs with prescribed Lick-White number. These results are used to give lower bounds on the genus of such graphs.
\end{abstract}

In 1948, B. Descartes [8] proved the existence of triangle-free graphs with chromatic number 4 . Since then, relationships between the chromatic number and girth have been investigated by a number of authors. Perhaps the most startling result is that proved, using different methods, by Erdös [9] and Lovász [16].

THEOREM A (ERDöS AND LovÁSz). For every pair of integers $k, t$, where $k \geqslant 2$, there is a graph of $G$ with $\chi(G)=k$ and $g(G) \geqslant t$, where $\chi(G)$ is the chromatic number and $g(G)$ is the girth of $G$.

More recently Lick and White [14] and [15] used the concept of $n$ degenerate graph in order to generalize chromatic number. For nonnegative integer $n$, a graph is called $n$-degenerate if each of its subgraphs has a vertex of degree at most $n$. For each $n$ the Lick-White number of graph $G$ is denoted by $\chi_{n}(G)$ and is defined as the least number of sets into which $V(G)$ can be partitioned such that each set induces an $n$-degenerate graph. Other papers which include results on Lick-White numbers include Simões-Pereira [21], [22], and [23], Cook [7], Jones [12] and Mitchem [18]. The chromatic number and point-arboricity of $G$ are respectively $\chi_{0}(G)$ and $\chi_{1}(G)$. For definitions and notation not defined in the papers see Harary [11].

A natural problem is to attempt to generalize results on girth and chromatic number to Lick-White numbers and girth. In [19] Mitchem constructed graphs with $g(G)=4$ and $f_{n}(G)$ arbitrarily large, where $f_{n}(G)$ is different from but analogous to $\chi_{n}(G)$. By very slightly modifying that work one obtains graphs with $g(G)=4$ and $\chi_{n}(G)$ arbitrarily large. Simões-Pereira [21] constructed much smaller graphs with the same property. In [7] Cook proves,

Received by the editors July 23, 1976 and, in revised form, April 15, 1977.

AMS (MOS) subject classifications (1970). Primary 05C15; Secondary 05C10.

$K e y$ words and phrases. $n$-degenerate graph, Lick-White number, genus. 
using the methods of Erdös:

THEOREM B. For every integer triple $n, k, t ; n \geqslant 0, k \geqslant 2, t \geqslant 1$, there is a graph $G$ with $\chi_{n}(G)=k$ and $g(G) \geqslant t$.

Cook's proof, unlike those of Simões-Pereira and Mitchem, shows existence, but does not actually construct the required graph. In this paper we use Theorem A to give a very short proof of Theorem B. Also, we will give a new lower bound, Theorem 9, which improves a result of Cook, on the genus of a triangle-free graph $G$ with $\chi_{n}(G)=k$. Catlin [4] recently gave a significant improvement of Brooks' Theorem for graphs with small clique number. In Theorem 2 we generalize Catlin's Theorem using Mitchem's [18] new generalization of Brooks' Theorem. Theorem 2 is used in the proof of Theorem 9. We also improve certain bounds given by Chvátal [5] and Kronk [13].

Borodin and Kostochka [3] as well as Bollobás and Thomason [1] have also proved Theorem 2. The former uses Borodin's version [2] of Theorem E. Theorem B is further improved in [1] where the existence is proved of a graph $G$ which is uniquely partitionable into $k n$-degenerate graphs and $g(G) \geqslant t$.

The maximum (minimum) degree of $G$ is denoted by $\Delta(G)(\delta(G))$. If $U \subset V(G)$, then $\langle U\rangle$ denotes the subgraph induced by $U$. The greatest integer function and least integer function are denoted respectively by $[x]$ and $\{x\}$. In order to give our proof of Theorem B, we need the following elementary results.

Theorem C (LICK AND WhITE [14]). $A$ graph $G$ is $n$-degenerate iff $V(G)$ can be ordered $v_{1}, v_{2}, \ldots, v_{p}$ such that in graph $\left\langle\left\{v_{i}, v_{i+1}, \ldots, v_{p}\right\}\right\rangle, \operatorname{deg} v_{i} \leqslant$ n.

THEOREM 1. For every $n \geqslant 1, \chi_{0}(G) /(n+1) \leqslant \chi_{n}(G) \leqslant \chi_{0}(G)$.

Proof. The second inequality is immediate. For the first inequality, let $\chi_{n}(G)=h$. Thus there is a partition $W_{1}, \ldots, W_{h}$ of $V(G)$ such that each $\left\langle W_{i}\right\rangle$ is $n$-degenerate. It follows from Theorem $\mathrm{C}$ that $\chi_{0}\left(\left\langle W_{i}\right\rangle\right) \leqslant n+1$ for each $i$. Hence, $\chi_{0}(G) \leqslant(n+1)\left(\chi_{n}(G)\right)$.

Proof of Theorem B. Consider $k^{\prime}=(n+1) k$. By Theorem A there is a graph $G$ with $g(G) \geqslant t$ and $\chi_{0}(G)=k^{\prime}$. By Theorem $1, \chi_{n}(G) \geqslant k$. Now remove sufficient points from $G$ until $G^{\prime}$ is obtained such that $\chi_{n}\left(G^{\prime}\right)=k$ and $g\left(G^{\prime}\right) \geqslant t$.

As did Catlin, we will use the following theorem.

THEOREM D (LOVÁSz [17]). For any positive integer $a$, if $h_{1}, \ldots, h_{a}$ are nonnegative integers such that $h_{1}+\cdots+h_{a}=\Delta(G)-a+1$, then there is a partition $V_{1}, \ldots, V_{a}$ of $V(G)$ such that $\Delta\left(\left\langle V_{i}\right\rangle\right) \leqslant h_{i}$ for $i=1,2, \ldots, a$.

Theorem E (Mitchem [18]). For $n \geqslant 0$, let $G$ be a connected graph other than 
(i) A complete graph with $t(n+1)+1$ vertices, $n \geqslant 0, t \geqslant 0$.

(ii) $A n(n+1)$-regular graph, $n \geqslant 0$.

(iii) An odd cycle, $n=0$.

Then $\chi_{n}(G) \leqslant\{\Delta(G) /(n+1)\}$.

Theorem E can be viewed as a natural analogue to Brooks' Theorem and will also be used to prove the next theorem, which shows that if a graph has small clique number then the bound given in Theorem $\mathrm{E}$ can be improved.

Theorem 2. Suppose $G$ is a graph with clique number $s$, and let $n$ be $a$ nonnegative integer. Also let $r$ be the smallest integer of the form $t(n+1)$, $t \geqslant 2$, such that $r \geqslant \max \{3, s\}$. Then $\chi_{n}(G) \leqslant\{(\Delta(G)+1-m) /(n+1)\}$ where $m=[(\Delta(G)+1) /(r+1)]$.

Proof. By Theorem E, if $m=0$ or 1 , Theorem 2 is true. Assume $m \geqslant 2$ and let $h_{i}=r$ for $i=1, \ldots, m-1$ and

$$
h_{m}=\Delta(G)+r+1-(r+1)[(\Delta(G)+1) /(r+1)] \geqslant r
$$

By Theorem $\mathrm{D}$, there exists a partition $V_{1}, \ldots, V_{m}$ of $V(G)$ such that $\Delta\left(\left\langle V_{i}\right\rangle\right) \leqslant r$ for $i=1, \ldots, m-1$ and $\Delta\left(\left\langle V_{m}\right\rangle\right) \leqslant \Delta(G)+(r+1)(1-m)$. Since $G$ has clique number $s \leqslant r$, no $\left\langle V_{i}\right\rangle$ contains $K_{r+1}$. According to Theorem E,

$$
\chi_{n}\left(\left\langle V_{i}\right\rangle\right) \leqslant\{r /(n+1)\}=t \text { for } i=1, \ldots, m-1
$$

and

$$
\chi_{n}\left(\left\langle V_{m}\right\rangle\right) \leqslant\{(\Delta(G)+(r+1)(1-m)) /(n+1)\} .
$$

It follows that

$$
\begin{aligned}
\chi_{n}(G) & \leqslant \chi_{n}\left(\left\langle V_{1}\right\rangle\right)+\cdots+\chi_{n}\left(\left\langle V_{m}\right\rangle\right) \\
& =(m-1) t+\{(\Delta(G)+1-t(n+1)(m-1)-m) /(n+1)\} \\
& =\{(\Delta(G)+1-m) /(n+1)\} .
\end{aligned}
$$

Corollary F (CATlin). If graph $G$ has clique number $s$, then $\chi_{0}(G) \leqslant \Delta(G)$ $+1-[(\Delta(G)+1) /(\max \{3, s\}+1)]$.

It should be noted that Grünbaum [10] asked that for every $k \geqslant 4$ does there exist a triangle-free graph $G$ with $\chi_{0}(G)=k=\Delta(G)$. Chvátal [6] has found the smallest such graph with $k=4$. Catlin's Theorem implies no such graph exists with $k \geqslant 7$.

Let $f_{n}(k)$ be the minimum number of vertices in a triangle-free graph $G$ with $\chi_{n}(G)=k$. It is transparent that $f_{0}(2)=2, f_{0}(3)=5$. Chvátal [5] showed that $f_{0}(4)=11$, and that the so-called Mycielski graph [20] is the only graph which obtains this minimum.

THEOREM 3. For $n \geqslant 0, f_{n}(2)=2(n+1)$.

Proof. The graph $G=K(n+1, n+1)$ shows that $f_{n}(2) \leqslant 2(n+1)$. 
Assume that $H$ is the smallest graph which has $\chi_{n}(H)=2$. Thus $\delta(H) \geqslant n$ +1 and $2 q \geqslant(n+1) p$ where $q$ and $p$ are the number of edges and vertices of $G$, respectively. However, by Turan's Theorem [24] any triangle-free graph has $2 q \leqslant p^{2} / 2$, and thus $p \geqslant 2(n+1)$.

We now give lower and upper bounds on $f_{n}(3)$. The upper bound is proved by constructing the smallest known triangle-free graph $G$ with $\chi_{n}(G)=3$.

THEOREM 4. For $n \geqslant 0, f_{n}(3) \leqslant(2 n+1) 5$.

Proof. Let $H_{1}$ be the 5-cycle $v_{1}, v_{2}, v_{3}, v_{4}, v_{5}$ and $\bar{H}_{2}=K_{2 n+1}$. Define $G$ as the composition $H_{1}\left[H_{2}\right]$. (See Harary [11].) Now $g(G)=4$. For $i=$ $1,2,3,4,5$ let $V_{i}$ be the set of vertices of $G$ with first coordinate $v_{i}$. The partition $V_{1} \cup V_{3}, V_{2} \cup V_{4}, V_{5}$ shows that $\chi_{n}(G) \leqslant 3$. Suppose that $V(G)$ can be partitioned into $n$-degenerate sets $R$ and $S$. At least $(n+1)$ points of $V_{1}$ are in one of the sets, say $R$. Thus at least $(n+1)$ points of $V_{2}$ are in $S$. It follows that at least $(n+1)$ points of $V_{4}$ are also in $S$. This implies that $S$ contains at most $n$ vertices of $V_{5}$, as does $R$, a contradiction.

Before proving Theorem 5, we state a needed definition and theorem. Graph $G$ is called $(n, k)$-critical if $\chi_{n}(G)=k$ and for every proper subgraph $H$ of $G, \chi_{n}(H)<k$.

THEOREM G (LICK AND WHITE). If $G$ is $(n, k)$-critical, then $\delta(G) \geqslant(n+$ $1)(k-1)$.

THEOREM 5. For $n \geqslant 1, f_{n}(3) \geqslant 6 n+7$.

Proof. Let $G$ be a triangle-free graph of minimum order with $\chi_{n}(G)=3$. By Theorems $G$ and $E$ we have that $\delta(G) \geqslant 2 n+2$ and $\Delta(G) \geqslant 2 n+3$. Let $v$ be a point of maximum degree with neighbor set $W=\left\{w_{1}, \ldots, w_{t}\right\}$, $t \geqslant 2 n+3$. Since $g(G)>3$, no two $w_{i}$ are adjacent. Let $B=V(G)-W-$ $\{v\}$. Since $\chi_{n}(\langle B\rangle) \geqslant 2,\langle B\rangle$ contains a path with at least $2 n$ vertices. Suppose there is a path $P=u_{1}, u_{2}, \ldots, u_{s}, s \leqslant 2 n$, such that $\langle B\rangle-P$ is $n$-degenerate. Then $(B-V(P)) \cup\{v\}, V(P) \cup W$ is a partition of $V(G)$ into two $n$-degenerate sets, a contradiction. It follows that there is a path $Q=v_{1}, \ldots, v_{2 n}$ in $\langle B\rangle$ such that $\chi_{n}(\langle B\rangle-Q)=2$. Thus there is a subgraph $H$ of $\langle B\rangle-Q$ with $\delta(H) \geqslant n+1$. Since $H$ is triangle-free, it has at least $2 n+2$ points. This implies that the order $p$ of $G$ is at least $6 n+6$. Suppose $p=6 n+6$, thus $H=\langle B\rangle-Q=K(n+1, n+1)$, so that $V(H)$ $=X \cup Y$ where every vertex of $X$ is adjacent with every vertex of $Y$. Any vertex of $W \cup V(Q)$ is adjacent with elements of only one of $X$ and $Y$. Also, each element of $W$ is adjacent to at most $n$ elements of $V(Q)$. Let $W_{x}=\left\{w_{i}\right.$ : $w_{i}$ is adjacent with no element of $\left.X\right\}$ and $W_{y}=\left\{w_{i}: w_{i}\right.$ is adjacent with no element of $Y\}$. Then $X_{1}=X \cup W_{x} \cup\left\{v_{1}, v_{2}, \ldots, v_{n}\right\}, X_{2}=Y \cup W_{y} \cup$ $\left\{v_{n+1}, \ldots, v_{2 n}\right\} \cup\{v\}$ yields a partition of $V(G)$ into $2 n$-degenerate sets. This contradiction implies that $f_{n}(3) \geqslant 6 n+7$.

THEOREM 6. For $n \geqslant 0, k \geqslant 4, f_{n}(k) \geqslant f_{n}(k-1)+k n+k+n-1+$ 
$k / t$, where $t=3$ if $n=0$, and $t=2$ otherwise.

Proof. Let $G$ have $\chi_{n}(G)=k$, deg $v=\Delta(G)$, and $W$ be the set of points adjacent with vertex $v$ of degree $\Delta(G)$ in $G$. Theorem 2 implies that $|W| \geqslant k n$ $+k-n-2+k / t$. Now $G-W-\{v\}$ contains an $(n, k-1)$-critical graph $H$ with $\delta(H) \geqslant(k-2)(n+1)$ and thus $H$ contains a path $P$ with $2 n$ points. Since $H$ is triangle-free, each point of $W$ is adjacent with at most $n$ points of $P$ and thus $\langle V(P) \cup W\rangle$ is $n$-degenerate. It follows that $\chi_{n}(G-P$ $-W-\{v\}) \geqslant k-1$. Thus $|G| \geqslant f_{n}(k-1)+k n+k-n-2+k / t+$ $2 n+1$.

Corollary 7. For $n \geqslant 1, k \geqslant 3$, and for $n=0, k \geqslant 5, f_{n}(k) \geqslant 4-3 n+$ $\left(k^{2}+k-12\right) / 2 t-k / 2+3 n k / 2+k^{2}(n+1) / 2$.

Proof. For $n \geqslant 1, k \geqslant 3$, the corollary follows immediately from the difference equation given in Theorem 6 together with Theorem 5. For $n=0$, $k \geqslant 5$ the corollary is implied by using $f_{0}(5) \geqslant 17$ from Chvátal [5] as our boundary condition and Theorem 6 .

Corollary 8. For $k \geqslant 5, f_{0}(k) \geqslant 2 k^{2} / 3-k / 3+2$.

In [5] Chvátal shows that $f_{0}(k) \geqslant(k+2)(k+1) / 2-4$ for $k \geqslant 4$. For $k \geqslant 7$, the bound given in Corollary 8 is better. Although there exist graphs with no triangles and arbitrarily high Lick-White numbers, Cook by Theorem $\mathrm{H}$ showed that such graphs necessarily have large genus.

THEOREM H (COOK [1]). Let $G$ be a graph of positive genus, $\gamma(G)$, with $g(G) \geqslant 4$. Then $\chi_{n}(G) \leqslant 1+[(2+2 \sqrt{\gamma(G)}) /(n+1)]$.

Theorem $\mathrm{H}$ can be viewed as a lower bound for $\gamma(G)$ in terms of $n$ and $\chi_{n}(G)$. Our final theorem improves Theorem $\mathrm{H}$.

THEOREM 9. Let $G$ be a triangle-free graph with $\chi_{n}(G)=k$ where $k \geqslant 3$ if $n \geqslant 1$, and $k \geqslant 5$ if $n=0$.

$$
\begin{aligned}
8 \gamma(G) \geqslant & (k n+k-n-5) \\
& \left(4-3 n+\left(3 n k+k^{2} n+k^{2}-k\right) / 2+\left(k^{2}+k-12\right) / 2 t\right) \\
& +k / t+7
\end{aligned}
$$

where $t=3$ if $n=0$ and $t=2$ if $n \geqslant 1$.

Proof. Since every graph with $\chi_{n}(G)=k$ contains a $(n, k)$-critical subgraph, it suffices to prove Theorem 9 for $(n, k)$-critical graphs. Let $G$ have $p$ points and $q$ lines. From Theorems $\mathrm{G}$ and 2 we have

$$
2 q \geqslant(k-1)(n+1) p+k / t-1 .
$$

However, from the generalized Euler formula

$$
2 q \leqslant 8 \gamma(G)+4 p-8
$$

it follows that 


$$
8 \gamma(G) \geqslant[(k-1)(n+1)-4] p+k / t+7 .
$$

The result follows by Corollary 7.

In [13] Kronk obtained a lower bound for $\gamma(G)$ in terms of $\chi_{n}(G)=k$ and $g(G)$, if $G$ is triangle-free. His bound is quadratic in $k$. Thus, for each value of $g(G) \geqslant 4$, there is a value $k_{0}$ such that if $\chi_{0}(G) \geqslant k_{0}$, then Theorem 9 gives a better bound than Kronk's.

\section{REFERENCES}

1. B. Bollobás and A. G. Thomason, Uniquely partitionable graphs (to appear).

2. O. V. Borodin, On decomposibition of graphs into degenerate subgraphs, Diskret. Analiz 28 (1976). (Russian)

3. O. V. Borodin and A. V. Kostochka, On an upper bound of the graph's chromatic number depending on graph's degree and density, Novosibirsk, Siberia (preprint).

4. P. Catlin, $A$ bound on the chromatic number of graphs (preprint).

5. V. Chvatal, The minimality of the Myscielski graph, Lecture Notes in Math., vol. 406, Springer-Verlag, Berlin and New York, 1974, pp. 243-246.

6. The smallest triangle-free 4-chromatic, 4-regular graph, J. Combinatorial Theory 9 (1970), 93-94.

7. R. J. Cook, Point partition numbers and girth, Proc. Amer. Math. Soc. 49 (1975), 510-514.

8. B. Descartes, A three-colour problem, Eureka 10 (1948), 24.

9. P. Erdös, Graph theory and probability, Canad. J. Math. 11 (1959), 34-38.

10. B. Grünbaum, A problem in graph coloring, Amer. Math. Monthly 77 (1970), 1080-1092.

11. F. Harary, Graph theory, Addison-Wesley, New York, 1969.

12. R. P. Jones, Hereditary properties and P-chromatic numbers, Combinatorics, London Math. Soc. Lecture Note Ser., vol. 13, Cambridge Univ. Press, London, 1974, pp. 83-88.

13. H. V. Kronk, The chromatic number of triangle-free graphs, Graph Theory and Applications, Lecture Notes in Math., vol. 303, Springer-Verlag, Berlin and New York, 1972, pp. 179-181.

14. D. R. Lick and A. T. White, $k$-degenerate graphs, Canad. J. Math. 12 (1970), 1082-1096.

15. The point-partition numbers of closed 2-manifolds, J. London Math. Soc. (2) 4 (1972), 577-587.

16. L. Lovász, On chromatic number of finite set-systems, Acta Math. Acad. Sci. Hungar. 19 (1968), 59-67.

17. __ On decomposition of graphs, Studia Sci. Math. Hungar. 1 (1966), 237-238.

18. J. Mitchem, An extension of Brooks' theorem to n-degenerate graphs, Discrete Math. 17 (1977), 291-298.

19. , On extremal partitions of graphs, Ph. D. Dissertation, Western Michigan Univ., 1970, pp. 103-109.

20. J. Mycielski, Sur le coloriage des graphes, Colloq., Math. 3 (1955), 161-162.

21. J. M. S. Simões-Pereira, $A$ note on graphs with prescribed clique and point-partition numbers, J. Combinatorial Theory Ser. B 14 (1973), 256-258.

22. , Joins of $n$-degenerate graphs and uniquely $(m, n)$-partitionable graphs, J. Combinatorial Theory Ser. B 21 (1976), 21-29.

23. On graphs uniquely partitionable into n-degenerate subgraphs, Coll. Math. Soc. Janos Bolyai, 10 Infinite and Finite Sets, Keszthely (Hungary), 1973.

24. P. Turan, On the theory of graphs, Colloq. Math. 3 (1954), 19-30.

Department of Mathematics, San Jose State University, San Jose, California 95192 